\title{
Heavy Baryons - Recent and Very New Results
}

Peter S. Cooper ${ }^{\text {a* }}$

${ }^{a}$ Fermi National Accelerator Laboratory

P.O. Box 500, MS 122, IL 60510

Recent results on observations, properties and decay modes of the charmed and beauty baryons will be reviewed. Candidates for several new high mass states which include a cleanly-identified daughter $\Lambda_{c}^{+}$baryon are seen in data from the SELEX experiment at Fermilab. These states are candidates for doubly-charmed baryons: a $\Xi_{c c}^{++}$ state and a $\Xi_{c c}^{+}$state. These candidates are more than $5 \sigma$ signals in each case at masses of 3520 and $3460 \mathrm{MeV}$ respectively.

\section{INTRODUCTION}

There have been several new results in heavy baryon physics since the last conference in this series two years ago. There are no new results on beauty baryons, due mainly the the absence of new data from the high energy colliders which can access these states. There are new decay modes and excited states seen in charmed baryons by CLEO and others which, for lack of space and time, I will omit here. I will focus on a set of new charmed baryon lifetime measurements by several experiments with precisions approaching $1 \%$ using contrasting experimental techniques and on the claimed discovery, by the SELEX experiment, of doubly charmed baryon states.

\section{CHARMED BARYON LIFETIMES}

Measurements of the lifetimes of the charmed baryons provide insight into the various diagrams contributing to charm decay. It has been long known from the differences of the lifetimes of the charged and neutral charmed mesons, that the simple spectator amplitude cannot dominate charmed hadron decay.

The qualitative hierarchy of the lifetimes of the 4 weakly decaying charm baryon states have been predicted long ago [1,2]. Measurements with sufficient precision to test these ideas quantitatively

\footnotetext{
*Presented at 5th International Conference on Hyperons, Charm, and Beauty Hadrons, Vancouver, BC, Canada, 25-29 July 2002.
}

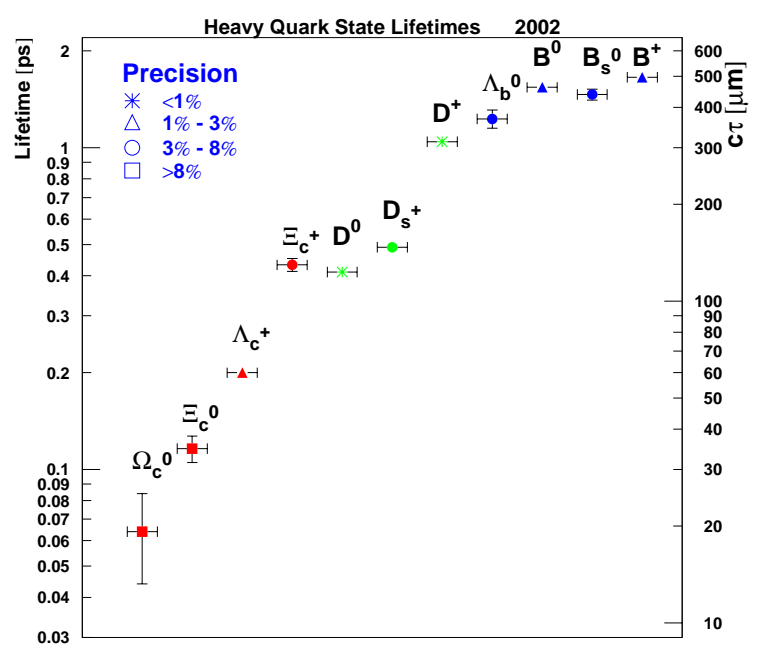

Figure 1. Heavy quark states lifetimes.

are now being made. As shown in Figure 1 the lifetimes of the weakly decaying charmed hadrons span a factor of 20 , in strong contrast with the lifetimes of the known $b$ hadrons. The question is "what physics processes cause these large lifetime differences?".

Three experiments have reported new charmed baryon lifetime measurements. SELEX and FO- 
CUS at Fermilab are fixed target charmed hadro and photo production experiments, respectively. They require a charmed baryon decay to fly several standard deviations and measure its lifetime with a reduced proper-lifetime analysis. CLEO produces charmed baryons in $e^{+} e^{-}$annihilation and analyses their lifetimes as a shift from zero in the flight length distributions. CLEO has a much cleaner background environment than possible in either photo or hadro-production.

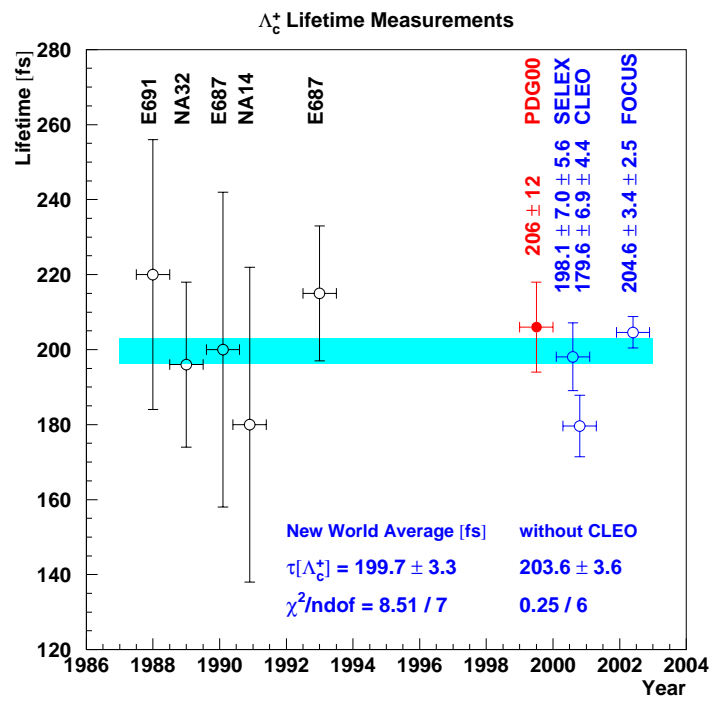

Figure 2. $\Lambda_{c}^{+}$lifetime measurements.

There are three new $\Lambda_{c}^{+}$lifetime measurements [3-5], each with an uncertainty smaller that the PDG-2000 world average (shown in Figure 2). The combined precision is approaching $1 \%$; however, there may be some systematic differences between the measurement techniques at the present level of precision. The new PDG average [6] assigns a scale factor of 1.6 in fitting these data.

At the $5 \%$ precision level there are two new $\Xi_{c}^{+}$ lifetime measurements $[7,8]$ (shown in Figure 3)
Table 1

New FOCUS $\Xi_{c}^{0}$ and $\Omega_{c}^{0}$ lifetimes [10].

\begin{tabular}{ccc}
\hline \hline State & Lifetime $[f s]$ & events \\
\hline$\Xi_{c}^{0}$ & $118 \pm 13 \pm 5$ & $110 \pm 17$ \\
$\Omega_{c}^{0}$ & $79 \pm 12 \pm 8$ & $64 \pm 14$ \\
\hline \hline
\end{tabular}

which agree well with each other but are somewhat above the older measurement from E687 [9]. The new PDG average [6] scale factor here is 1.3.

The FOCUS experiment also reported new measurements at this conference [10] for both the $\Xi_{c}^{0}$ and $\Omega_{c}^{0}$ shown in Table 1. Each is more precise, and above, the previous world data.

These precision lifetime measurements are reaching the point where a meaningful quantitative comparison with models is required.

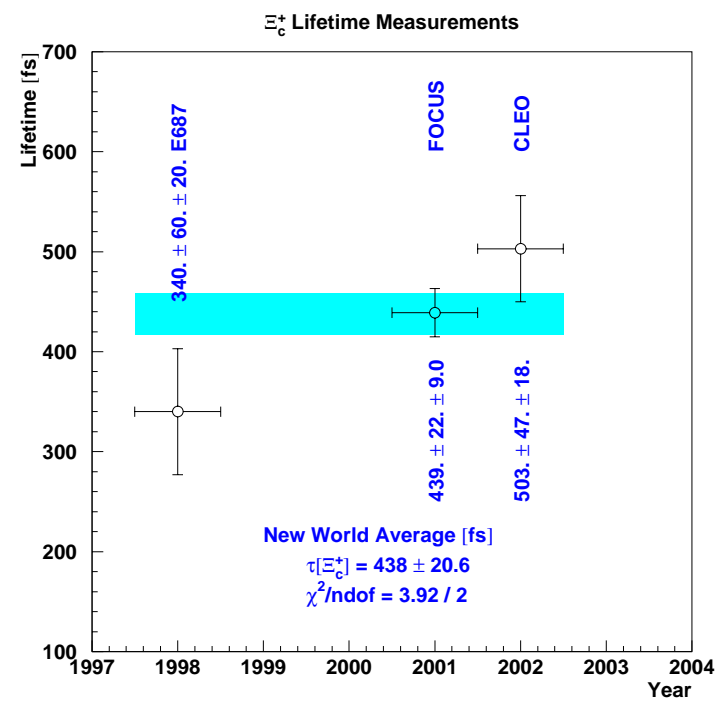

Figure $3 . \Xi_{c}^{+}$lifetime measurements. 


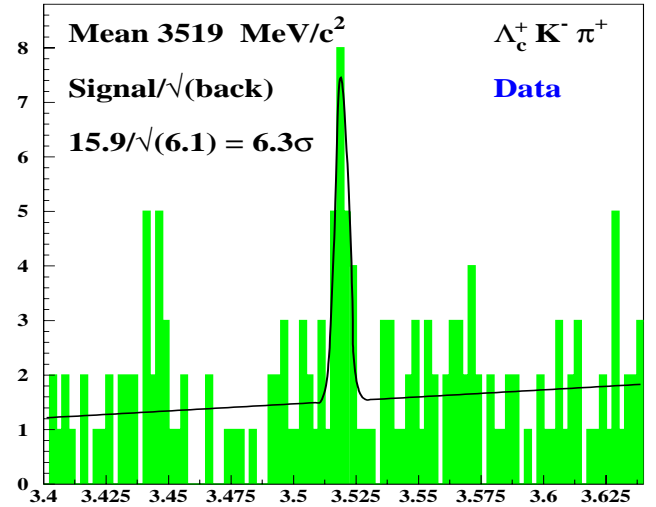

Figure 4. SELEX $\Xi_{c c}^{+}(3519)$ signal.

\section{DOUBLY CHARMED BARYON CANDIDATES FROM SELEX}

The SELEX experiment, of which I am a spokesman, reports evidence for three new high mass states decaying into $\Lambda_{c}^{+} K^{-} \pi^{+}\left(\pi^{+}\right)$. These states are consistent with some expectations of the doubly-charmed baryons predicted by the broken SU(4) symmetry of a system with four quark flavors. This work is presently being published in the refereed journals with one paper already in print [12] and others in preparation. I will only briefly summarize this work here.

SELEX reports a $\Xi_{c c}^{+}$state at $3519 \mathrm{MeV}$ with a statistical significance of $6.3 \sigma$ (Figure 4 ) and an apparent iso-partner $\Xi_{c c}^{++}$state at $3460 \mathrm{MeV}$ with a statistical significance of $4.8 \sigma$ (Figure 5 ). Both of these states have a width consistent with resolution and a very short lifetime ( $<30 \mathrm{fs})$. SELEX also sees a broad state at $3783 \mathrm{MeV}$ (Figure 6) which looks like a $\Xi_{c c}^{*++}$ with evidence for the strong decay mode $\Xi_{c c}^{*++}(3783) \rightarrow \Xi_{c c}^{+}(3519) \pi^{+}$.

The FOCUS experiment showed at this conference [13] their search for states like SELEX observes. Starting with 10 times more $\Lambda_{c}^{+}$events they have no evidence for any signals, and few entries in general, in a long list of decay modes, including those where SELEX see signals. What-

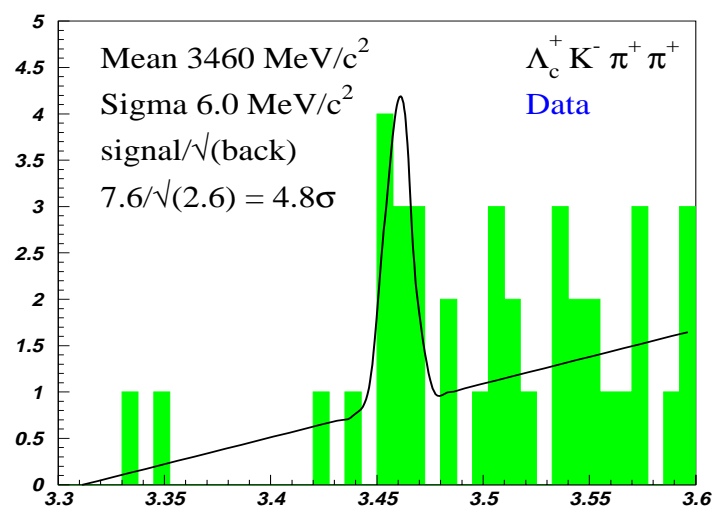

Figure 5. SELEX $\Xi_{c c}^{++}(3460)$ signal.

ever SELEX observes in hadro-production with baryon beams is not seen in photo-production.

The SELEX signals do not constitute a clear and unambiguous picture as doubly charmed baryons. The production cross-section of these states, in the forward region where SELEX looks, is considerably higher than expected; lifetimes as short as $30 \mathrm{fs}$ are less than models predict and a $60 \mathrm{MeV}$ mass difference between $\Xi_{c c}^{+}(3519)$ and $\Xi_{c c}^{++}(3460)$ is hard to understand as an iso-spin splitting.

\section{FUTURE PROSPECTS IN HEAVY BARYON PHYSICS}

Heavy baryon physics is still an active field. Analyses continue from data taken by SELEX, FOCUS CLEO and Fermilab E791. Both CDF and D0 at at the Tevatron Collider are now collecting large new samples of both charm and beauty baryons. The COMPASS experiment at CERN has begun running with plans for a significant charm physics program. Belle is already reporting charmed baryon results[11]. Babar will surely enter this game too. The CLEO-C upgrade plans at Cornell don't mention charmed baryons but, given their long and successful charmed baryon program at CLEO, I would be surprised if there were not new charmed baryon results from them too. 


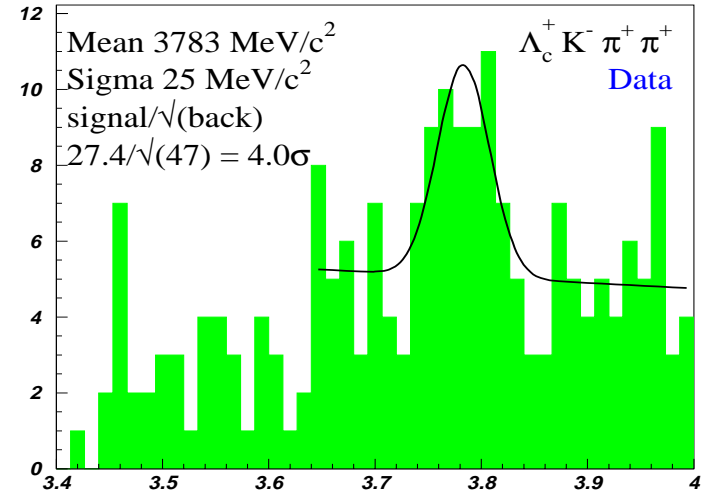

Figure 6. SELEX $\Xi_{c c}^{*++}(3783)$ signal.

We can look forward, in the next edition of this conference, to further measurements from SELEX, and hopefully others, to confirm (or contradict) their signals. New work on the phenomenology and models of doubly charmed baryons can also be expected in order to more fully understand these observations.

We can expect be hear new and exciting results in heavy baryon physics at Beach2004.

\section{REFERENCES}

1. B. Guberina, R. Ruckl and J. Trampetic, Z. Phys. C 33, 297 (1986).

2. M. A. Shifman and M. B. Voloshin, Sov. Phys. JETP 64, 698 (1986) [Zh. Eksp. Teor. Fiz. 91, 1180 (1986)].

3. SELEX Collaboration, A. Kushnirenko et al., Phys. Rev. Lett. 86 (2001) 5243.

4. CLEO Collaboration, A.H. Mahmood et al., Phys. Rev. Lett. 86 (2001) 2232.

5. FOCUS Collaboration, J. Link et al., Phys. Rev. Lett. 88 (2002) 161801.

6. Particle Data Group, K. Hagiwara et al., Phys. Rev. D 66 (2002) 010001.

7. FOCUS Collaboration, J. Link et al., Phys. Lett. B 523 (2001) 53.

8. CLEO Collaboration, A.H. Mahmood et al., Phys. Rev. D 65 (2002) 031102.

9. E687 Collaboration, P.L. Frabetti et al.,
Phys. Lett. B 427 (1998) 211.

10. FOCUS Collaboration, presented by E. Vaandering, this conference.

11. K. Abe et al., Phys. Lett. B 524, 33 (2002)[arXiv:hep-ex/0111032].

12. SELEX Collaboration, M. Mattson et al., Phys. Rev. Lett. 89 (2002) 112001.

13. FOCUS Collaboration, presented by S. Ratti, this conference. 